\title{
The Impact of an ICU Liaison Nurse on Discharge Delay in Patients after Prolonged ICU Stay
}

\author{
W. CHABOYER*, L. THALIB $\dagger$, M. FOSTER $\$$, D. ELLIOTT\&, R. ENDACOTT ${ }^{* *}$, B. RICHARDS $\dagger \dagger$ \\ Griffith University, Gold Coast Campus, Queensland
}

\begin{abstract}
SUMMARY
The mismatch between intensive care unit (ICU) bed availability and demand may be improved with timely patient discharges, however little is known about the nature and contributing factors of discharge delays. This study investigated the impact of a specific intervention - the ICU liaison nurse role - in reducing ICU discharge delay using a prospective block intervention study. One hundred and eighty-six ICU patients (101 control and 85 liaison nurse intervention) with an ICU length of stay of three days or longer and who survived to ICU discharge were examined. The liaison nurse was involved in assessment of patients for transfer to the ward, with a major focus on coordinating patient transfer including liaison with ward staff prior to and following ICU discharge. Logistic regression was used to quantify the risk of discharge delay associated with the liaison nurse intervention with adjustment for potential confounding variables. While no demographic or clinical variables were significant predictors of ICU discharge delay, those in the liaison nurse group were almost three times less likely to experience a discharge delay of at least two hours and about 2.5 times less likely to experience a delay of four or more hours. The positive effect of the liaison nurse role in reducing the discharge delay remained after adjusting for potential confounders. We conclude that the liaison nurse role is effective in reducing the discharge delay in ICU transfer.
\end{abstract}

Key Words: intensive care, discharge delay, follow-up, liaison nurse, outreach services

Intensive Care Unit (ICU) beds are both an expensive and a scarce resource in many hospitals ${ }^{1-3}$ comprising between 1.5 and $4 \%$ of total hospital beds in Australia and New Zealand ${ }^{4}$. A recent report identified that in 2001 there were 1,803 ICU beds in Australia (9.2 ICU beds/100,000 population) ${ }^{5}$. Given that the proportion of critically ill patients in hospitals is escalating ${ }^{6}$, it follows that ICU beds are a commodity in high demand ${ }^{7}$. With occupancy rates often as high as 80 to $90 \%{ }^{8-10}$, many critically ill patients who would benefit from ICU treatment may not be admitted in a

*R.N., Ph.D., Professor, Research Centre for Clinical Practice Innovation, Griffith University Gold Coast Campus, Bundall, Queensland.

$\dagger$ B.Sc., Ph.D., Associate Professor, Faculty of Medicine, Kuwait University, Kuwait.

$\ddagger$ R.N., M.N., Nurse Practice Coordinator, Gold Coast Hospital, Queensland.

$\S R . N .$, Ph.D., Professor, Department of Clinical Nursing, The University of Sydney, New South Wales.

**R.N., Ph.D., Professor, La Trobe University Bendigo and La Trobe/Alfred Clinical School, The Alfred Hospital, Melbourne, Victoria.

$\dagger \dagger$ M.B., B.S., F.R.A.C.P., F.J.F.I.C.M., Doctor, Executive Director Surgery and Critical Care, Director ICU, Gold Coast Hospital, Queensland.

Address for reprints: Professor Wendy Chaboyer, Research Centre for Clinical Practice Innovation, Griffith University Gold Coast Campus, PMB 50 Gold Coast Mail Centre, Bundall, Qld 9726.

Accepted for publication on August 8, 2004

Anaesthesia and Intensive Care, Vol. 34, No. 1, February 2006 timely fashion ${ }^{11-13}$, with a variety of factors influencing $\operatorname{admission}^{14-16}$.

Shortage of available beds may result in surgeries being cancelled ${ }^{10,17}$, referrals refused ${ }^{10,15}$, and critically ill patients transported to other hospitals ${ }^{3,10,18}$. The factors that impact on ICU bed availability are varied. Staffing ${ }^{9,17,19}$, admission and discharge decisions $^{2,9,20,17,14,21}$, open versus closed ICUs ${ }^{22,23}$, seasonal peaks in ICU bed demand and the presence of high

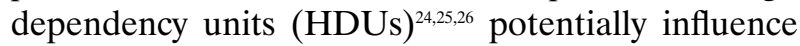
occupancy rates. While premature discharge has received some attention ${ }^{2,9,10,11}$, one factor that is rarely considered in the study of occupancy rates is the impact of discharge delay time on subsequent ICU bed availability. This time is defined as the interval between when patients are deemed clinically fit for discharge from ICU and when they actually vacate the bed $^{27}$. While only one published report in adult ICU could be found ${ }^{28}$, discharge delay was the focus of a two year study conducted in a neonatal $\mathrm{ICU}^{27}$. Despite a coordinated approach to ICU discharge, 116 neonates experienced a discharge delay that accounted for 480 patient days in this study. These delays were attributed to organizational factors beyond the ICU, family issues and lack of discharge planning ${ }^{27}$.

The ICU liaison nurse role ${ }^{29-32}$ is an emerging 
innovation that has a number of potential benefits including impact on ICU discharge delay. One aspect of the liaison nurse role relates to co-ordinating efficient patient transfer ${ }^{29,32,33}$. Activities such as communicating with receiving ward staff, assessing ward staff resources and skill-mix ${ }^{34,35}$, preparing both the ICU and ward staff for patient transfer, and assessing bed status may be encompassed within the transfer process. Another responsibility identified is continued facilitation of links between the ICU and ward staff after patient transfer ${ }^{29,33,34}$. It is these tasks that are likely to minimize discharge delay, however no evidence is currently available to support this proposition $^{36}$. Therefore, the purpose of this study was to examine the impact of an ICU liaison nurse on ICU discharge delay.

\section{PATIENTS AND METHODS}

A prospective block intervention research study was conducted in one adult Australian ICU. A randomized controlled trial was not possible due to the potential for contamination across groups. Each block was four months in duration; after the first control and intervention periods, a one-month washout period was followed by a second block of control and intervention periods. Four months for each block was chosen: 1) to ensure the intervention was in place for a reasonable duration in case the impact of the liaison nurse service was gradual; and 2) to account for seasonal variation. The wash-out period was included to examine whether factors other than the liaison nurse, such as education, could have been responsible for improvements in delay. The wash-out was kept relatively short to limit the likelihood of other historical and organisational factors confounding the study. Similar to Perlmutter ${ }^{27}$, discharge delay was defined as the time period from when the patient was deemed medically ready for discharge to when the patient actually left the ICU. Delay was assessed in two ways: a delay of at least two hours and a delay of at least four hours. Each of these periods is important from an organisational and staffing perspective. There were no changes in ICU admission or discharge policy during the study period. The study received approval from university and hospital ethics committees, including access to the clinical database used in this analysis.

\section{Sample and Setting}

The study was conducted at a 580-bed tertiary referral hospital with a 13-bed ICU that admits approximately 900 patients per year. Paediatric, burns and cardiac surgery patients were not admitted to the unit. The ICU clinical database was accessed to identify all patients who had an ICU length of stay (LOS) of three days or greater and who survived to ICU discharge. Prolonged stay patients were targeted to avoid relatively short stay postoperative patients with pre-planned admissions and discharges. Patients who died in ICU were excluded from the study.

\section{Intervention}

The ICU liaison nurse role was developed following a review of the literature and focus groups conducted with ICU survivors, their families, and both ICU and ward nurses. The liaison nurse was involved in assessment of patients for transfer to the ward, with a major focus being the coordination of ICU patient transfer and liaison with ward staff but was not involved in the final decision to discharge the patient from ICU. Tasks such as communicating with ward staff, assessing ward staff skill-mix and resources, preparing both the ICU and ward staff for patient transfer, and assessing bed status were encompassed in this activity. Other aspects included providing clinical support and resources to ward nurses in the capacity as educator of these staff in preparation for receiving ICU patients. The liaison nurse also provided practical and emotional support and education to patients and their families before and after discharge.

As the liaison nurse worked eight-hour days from Monday to Friday, part of her role included handover to a part-time liaison nurse who dedicated four hours each on Saturday and Sunday to the role, thus 1.2 full-time equivalents liaison nurse services in total. The full-time week day liaison nurse undertook most of the preparation and liaison for patients who were expected to be discharged during the weekend, to ensure the part-time weekend liaison nurse could concentrate predominantly on those patients who were unexpectedly being transferred. During the week, the liaison nurse was notified during usual working hours of patients who were expected to be transferred during the evening/night. This enabled preparation, assessment and liaison to be undertaken by the liaison nurse.

\section{Data Collection}

All data, excluding the presence or absence of the liaison nurse, were routinely collected by a data manager who maintained a clinical database developed by the Australian and New Zealand Intensive Care Society $^{37}$. The data manager was independent of the research team and blinded to the study block. That is, the data manager was aware that a liaison nurse had been hired but did not know that the liaison nurse 
provided services on some occasions (intervention blocks) but not others (control blocks). The actual database had a number of quality management features with both monthly audits and hand checks routinely performed to ensure accuracy of data entry. Collection of ICU discharge delay information had been part of the data collected prior to the start of the current study. The database was accessed to obtain data and admission dates were matched to the presence or absence of the liaison nurse. Discharge date was recoded to be either weekday or weekend and discharge time was recoded to be either in office hours' or 'after hours' defined as $2200-0659^{1}$. Discharge delay time was recorded in half-hour time increments. To test reliability, a second person also collected discharge delay data on 20 patients. The correlation between the data manager and the reviewer was highly significant $(\mathrm{r}=0.99, P<0.001)$ with only two recordings differing by one half-hour each.

\section{Data Analysis}

Clinical and patient characteristics were compared between the control and intervention group prior to estimating the risk of discharge delay associated with the liaison nurse intervention. Continuous variables were compared using Mann-Whitney $U$ test and categorical variables were compared using Z-test of proportion. The results are reported as median and inter-quartile range or frequency and percentage as appropriate. Logistic regression was used to calculate the odds ratios and 95\% confidence intervals for delays of at least two hours and four hours, with and without adjusting for factors that were thought to be potential confounders. Potential confounders that were assessed included age, gender, weekend/ weekday discharge, after-hours discharge, ICU LOS and surgical or medical and emergency or elective admissions. All data analyses were carried out using SPSS (SPSS Inc.USA).

\section{RESULTS}

A total of 188 patients were admitted to the study with two patients excluded from the analysis as information on their hours of delay was missing. This left 186 patients in the study, 101 in the control and 85 in the intervention group. Table 1 compares the demographic and clinical characteristics of the intervention with the control groups. The two groups were similar in age, APACHE II and estimated risk of death (ROD) scores. The gender distribution was similar in both groups and reflected the gender distribution for all patients at the study site in 2002.
There were no differences between the groups in proportion of emergency admissions, surgical versus medical admission, ICU readmissions or in hospital mortality. Weekend discharge or after-hours discharge or night discharge when the liaison nurse was not available were not found to be different between intervention and control groups. Hours of delay were significantly higher in the control group while ICU LOS was significantly higher in the intervention group. A further analysis of this ICU LOS using the MannWhitney revealed that the differences were significant in the first block but not in the second, suggesting that increase in ICU LOS in the study group is not consistent and most probably due to chance. There was no difference in hospital LOS nor was there a difference in the LOS on the ward alone (i.e. hospital LOS minus ICU LOS).

TABLE 1

Demographic and clinical characteristics of the participants $(N=186)$

\begin{tabular}{llll}
\hline & $\begin{array}{l}\text { Control } \\
\text { Group } \\
(\mathrm{N}=101) \\
\text { Median (IQR) }\end{array}$ & $\begin{array}{l}\text { Intervention } \\
\text { Group } \\
\text { Median }(\mathrm{IQR})\end{array}$ & \\
\hline Age (years) & $57(25.5)$ & $56(30.0)$ & $\mathrm{NS}$ \\
$\begin{array}{l}\text { ICU Length of Stay } \\
\quad \text { hours) }\end{array}$ & $135(116.5)$ & $169(136.5)$ & $*$ \\
Hospital Length of Stay & & & \\
$\quad$ (days) & $21(20.0)$ & $22(31.5)$ & $\mathrm{NS}$ \\
APACHE II Score & $17(10.0)$ & $18(9.0)$ & $\mathrm{NS}$ \\
Estimated ROD & $0.22(0.41)$ & $0.26(0.29)$ & $\mathrm{NS}$ \\
Hours of Delay & $2.0(5.0)$ & $0.0(2.0)$ & $* * *$ \\
\hline & $\mathrm{n}(\%)$ & $\mathrm{n}(\%)$ & \\
\hline Male & $59(58.4)$ & $50(58.8)$ & $\mathrm{NS}$ \\
$>2$ hours delay & $49(49.0)$ & $19(22.4)$ & $* *$ \\
$>4$ hours delay & $29(29.0)$ & $12(14.1)$ & $* * *$ \\
Emergency Admission & $92(91.1)$ & $77(90.6)$ & $\mathrm{NS}$ \\
Surgical admissions & $32(31.7)$ & $26(30.6)$ & $\mathrm{NS}$ \\
Weekend discharge & $20(19.8)$ & $15(17.6)$ & $\mathrm{NS}$ \\
ICU readmission & $4(4.0)$ & $2(2.4)$ & $\mathrm{NS}$ \\
After hours discharge & $17(16.8)$ & $19(22.4)$ & $\mathrm{NS}$ \\
Weekend discharge & $20(19.8)$ & $15(17.6)$ & $\mathrm{NS}$ \\
Died in hospital & $8(7.9)$ & $2(2.4)$ & $\mathrm{NS}$ \\
\hline NS: & & \\
\hline
\end{tabular}

NS: not significant; $* P<0.05 ; * * P<0.01 ; * * * P<0.001$.

Table 2 provides crude and adjusted odds ratio estimates of two or more hours of delay and four or more hours of discharge delay associated with the liaison nurse intervention. Overall, there were 68 $(36.6 \%)$ patients who experienced a discharge delay of at least two hours and $41(22.0 \%)$ experienced a discharge delay of more than four hours in the entire sample. Risk of a discharge delay of at least two hours were 3.2 times more in the control group compared to the liaison nurse intervention group when unadjusted for potential confounding variables. When these odds ratio estimates were adjusted for factors such as age, gender, time of discharge and ICU LOS, 
there were no noticeable differences in the estimate or their confidence interval. A longer delay of four hours or more had similar findings with no effect by potential confounders, but odds ratio estimates were slightly reduced to about 2.5 . All the odds ratio estimates demonstrated the prominent effect of the liaison nurse role in reducing discharge delay. When the adjustments were conducted with more than one factor, the odds ratio estimates remained consistently similar, indicating that the liaison nurse intervention was an independent contributor in significantly reducing discharge delays from ICU.

TABLE 2

Crude and adjusted risk estimates (Odds ratio and 95\% CI) of the effect of liaison nurse intervention nurses on the ICU discharge delay of 2 hours and 4 hours

\begin{tabular}{lll}
\hline & \multicolumn{2}{c}{ Discharge Delay } \\
\cline { 2 - 3 } & $\begin{array}{l}>2 \text { hours* } \\
\mathrm{n}=68\end{array}$ & $\begin{array}{l}>4 \text { hours** } \\
\mathrm{n}=41\end{array}$ \\
& $\mathrm{OR}(95 \% \mathrm{CI})$ & $\mathrm{OR}(95 \% \mathrm{CI})$ \\
\hline Unadjusted estimate & $3.3(1.7-6.2)$ & $2.5(1.2-5.2)$ \\
Age adjusted & $3.3(1.7-6.2)$ & $2.4(1.2-5.2)$ \\
Gender adjusted & $3.3(1.7-6.4)$ & $2.5(1.2-5.3)$ \\
Age and Gender & $3.3(1.7-6.4)$ & $2.5(1.2-5.3)$ \\
Weekend/weekday discharge & & \\
$\quad$ adjusted & $3.4(1.8-6.5)$ & $2.5(1.2-5.2)$ \\
After hours discharge adjusted & $3.4(1.8-6.5)$ & $2.8(1.3-6.1)$ \\
Elective admission adjusted & $3.3(1.7-6.2)$ & $2.4(1.2-5.2)$ \\
Surgical admissions adjusted & $3.3(1.7-6.2)$ & $2.5(1.2-5.2)$ \\
Age, gender, elective, and & & \\
$\quad$ surgical admission adjusted & $3.3(1.7-6.4)$ & $2.5(1.2-5.3)$ \\
ICU LOS adjusted & $3.3(1.7-6.3)$ & $2.5(1.2-5.3)$ \\
\hline
\end{tabular}

*All OR estimates are highly significantly different from unity at $P<0.001$.

**All OR estimates are significantly different from unit $P<0.05$.

\section{DISCUSSION}

A delay in discharge increases ICU length of stay, which can result in reduced ICU bed availability, inefficiency, and an unnecessary use of a costly and high demand commodity. This study evaluated the impact of a liaison nurse in reducing discharge delay. Results indicate that patients whose discharge did not involve a liaison nurse were 2.5 times more likely to experience a delay of four hours or longer than those who were discharged when the liaison nurse was involved. In fact, the existence of the liaison nurse was significantly related to a reduction in discharge delay, despite a significantly longer ICU LOS among the liaison nurse intervention group and despite controlling for a number of other factors. Other clinical factors that were likely to affect ICU patient discharge to the wards, namely discharge time, surgical status and elective or emergency admission status, did not demonstrate a significant effect. Furthermore, the APACHE II score, as a measure of severity of illness, was not a significant predictor of discharge delay.

While the reason that ICU LOS differed between the two groups is unknown, it is possible that the liaison nurse actually influenced these findings through systematic assessment of patient status prior to discharge. However, this ICU LOS difference was only evident in the first block so it is possible that this difference is due to chance. Alternatively the study numbers may have been too small to detect a higher severity of illness in the intervention group which may have also accounted for longer ICU LOS. Further examination of these assessment and related liaison activities is required to consider any effect on ICU LOS.

In particular, delays of four hours or more have significant implications for nursing staff, for effective service provision and for the welfare of the patient. Because these patients are essentially ward patients, and of a lower dependency, one of two workload issues may arise. ICU nurses may have to care for these patients in addition to their normal patient load or they may care for only the patient awaiting transfer. In the first instance, assuming that the normal workload is optimal, nurses are taking on additional work. In the second instance ICU nurses, who are in short supply ${ }^{18}$ are not being used optimally.

Discharge delay impacts on service efficiency, particularly when there is pressure on beds for new ICU admissions. It is possible that patients who require ICU beds may be admitted in a more timely fashion if decreasing discharge delay results in beds becoming available sooner. However, this proposition relies on two components: 1) ward patients are identified as needing ICU beds; and 2) they are not admitted to ICU in a timely fashion. Some research indicates that this indeed may be the case ${ }^{11-13}$, however the evidence is not conclusive. It is also worth mentioning that although our study indicated the role of liaison nurse in reducing the discharge delay, it is also important to identify the causes for delay. Further examination of the factors that influenced ICU discharge delay is needed.

Although this study demonstrated benefits to the liaison nurse role, several limitations are acknowledged. First, a block intervention design was used as the most pragmatic and powerful design available. It is however possible that the two groups differed on some variable that was not measured in the study. A randomized controlled trial (RCT) was not possible in this case because there was no way to eliminate contamination across groups within one institution, and a cluster RCT was not feasible because the cost of such a study would have been prohibitive ${ }^{45,46}$. 
In addition, actual reasons for the delay in this study were not recorded. It is not known if the delays were preventable irrespective of the presence of the liaison nurse. However, as Goldfrad and Rowan ${ }^{1}$ note, reasons for delay are subjective in nature and may be biased. A more objective measure of 'readiness for discharge' such as the Patient at Risk (PAR) scoring system $^{12}$ may be helpful in distinguishing between patients who are physiologically stable enough to be discharged and those who are simply less critically ill than the patient waiting to be admitted to the intensive care bed. While there is a growing body of knowledge in relation to discharge decision making ${ }^{2,9,14,7,2,21}$, the effect of discharge scoring systems on patient outcomes is not well understood.

The work hours of the liaison nurse are a consideration when attempting to improve discharge delay and continuity of care. While there was a fulltime liaison nurse during the weekdays, on weekends a part-time liaison nurse undertook this role. Thus, it is possible that weekend service differed to the weekday service. However, there were clear written guidelines for the weekend liaison nurse to follow, the weekday liaison nurse provided a 'handover' and the weekend liaison nurse did not have a clinical load during the hours worked as a liaison nurse. It may also be appropriate to have the liaison nurse working evening shifts and weekends, when there are less staffing resources in the ward areas. To note, these variables were used to adjust the odds ratio estimates and found not to be important in determining the level of discharge delays.

Finally, due to the sample size, we were not able to assess the effect of the liaison nurse on longer ICU discharge delay times such as eight or ten hours of delay. Because these delays were relatively rare, this study lacked the power to detect effects associated with longer delay times. Nonetheless, it was clear that a decrease in delays was clearly associated with the liaison nurse intervention.

Extending services to critically ill patients outside of the walls of the ICU is becoming increasingly common prior to ICU admission and now after ICU discharge $^{6,32}$, and intensive care services are thus more viewed within the operational context of the entire organisation, rather than an isolated unit. As such costs and benefits of the liaison nurse needs to be considered from this continuum of care perspective, and resultant cascade effects throughout the hospital. As well as improving resource utilisation within the ICU, there may be tangible flow on effects to other areas such as the Operating Theatre and Emergency Department are likely (including fewer delayed or cancelled theatre cases, and fewer critically ill emergency department patients delayed or refused ICU admission) and reduction in the re-admission to ICU as well as the less tangible but equally important decrease in stress felt by patients, staff and relatives by achieving timely and supported ward transfer.

This study has demonstrated that the ICU liaison nurse can impact on delay time in patients who have a prolonged ICU stay. In order to limit ICU discharge delay, a broad hospital wide perspective will also need to be considered in any future service enhancements. Future evaluation of liaison nurse roles should include examination of the reasons for discharge delay and ICU exit block in order to verify the findings presented here.

\section{ACKNOWLEDGEMENTS}

Funding to undertake this study was obtained from the Australian Research Council and the Gold Coast Hospital Intensive Care Trust.

\section{REFERENCES}

1. Goldfrad C, Rowan K. Consequences of discharges from intensive care at night. Lancet 2000; 355:1138-1142.

2. Daly K, Beale R, Chang RW. Reduction in mortality after inappropriate early discharge from intensive care unit: logistic regression triage model. BMJ 2001; 322:1274-1276.

3. Duke G, Green J. Outcome of critically ill patients undergoing interhospital transfer. Med J Aust 2001; 174:122-125.

4. Anderson T, Hart G. Review of intensive care activity 2000/2001. Australian and New Zealand Intensive Care Society, Melbourne 2002.

5. Anderson T, Hart G. Review of intensive care activity 1999/2000. Australian and New Zealand Intensive Care Society, Melbourne 2001.

6. Hillman K. Critical care without walls. Curr Opin Crit Care 2002; 8:594-599.

7. Ridley SA, Burchett K, Burns A, Gunning K. A comparison of hospital and critical-care activity. Anaesthesia 1999; 54:521528.

8. Richards B, Foster M. Gold Coast Hospital Department of Intensive Care 2002 Annual Report.

9. Zimmerman J, Wagner D, Draper E, Knaus W. Improving intensive care unit discharge decisions: Supplementing physician judgement with predictions of next day risk for life support. Crit Care Med 1994; 22:1373-1384.

10. Smith GB, Taylor BL, McQuillan PJ, Nials E. Rationing intensive care-intensive care provision varies widely in Britain. BMJ 1995; 310:1412-1413.

11. McQuillan P, Pilkington S, Allan A et al. Confidential inquiry into quality of care before admission to intensive care. BMJ 1998; 316:1853-1858.

12. Goldhill DR, Worthington L, Mulcahy A, Tarling M, Sumner A. The patient-at-risk team: identifying and managing seriously ill ward patients. Anaesthesia 1999; 54:853-860.

13. Hillman K, Parr M, Flabouris A, Bishop G, Stewart A. Redefining in-hospital resuscitation: the concept of the medical emergency team. Resuscitation 2001; 48:105-110. 
14. Sprung CL, Geber D, Eidelman LA et al. Evaluation of triage decisions for intensive care admissions. Crit Care Med 1999; 27:1073-1079.

15. Joynt GM, Gomersall CD, Tan P, Lee A, Cheng CA, Wond EL. Prospective evaluation of patients refused admission to an intensive care unit: triage, futility and outcome. Intensive Care Med 2001; 27:1459-1465.

16. Skowronski GA. Bed rationing and allocation in the intensive care unit. Curr Opin Crit Care 2001; 7:480-484.

17. Metcalfe MA, Sloggett A, McPherson K. Mortality among appropriately referred patients refused admission to intensivecare units. Lancet 1997; 350:7-11.

18. Dobb G. Bed availability and transfer of critically ill patients. Med J Aust 2001; 174:114-115.

19. Williams G, Clarke T. A consensus driven method to measure the required number of intensive care nurses in Australia. Aust Crit Care 2001; 14:106-115.

20. Society of Critical Care Medicine. Consensus statement on the triage of critically ill patients. JAMA 1994; 271:1200-1204.

21. Moreno R, Agthé D. ICU discharge decision-making: are we able to decrease post-ICU mortality? Intensive Care Med 1999; 25:1035-1036.

22. Carson SS, Stocking C, Podsadecki T et al. Effects of organizational change in the medical intensive care unit of a teaching hospital: a comparison of 'open' and 'closed' formats. JAMA 1996; 276:322-328.

23. Multz AS, Chalfin DB, Samson IM et al. A 'closed' medical intensive care unit (MICU) improves resource utilization when compared with an "open” MICU. Am J Resp Crit Care Med 1998; 157:1468-1473.

24. Garfield M, Jeffrey R, Ridley S. An assessment of the staffing level required for a high-dependency unit. Anaesthesia 2000; 55:137-143.

25. Boots R, Lipman J. High dependency units: issues to consider in their planning. Anaesth Intensive Care 2002; 30:348-354.

26. Coggins RP. Delivery of surgical care in a district general hospital without high dependency unit facilities. Postgrad Med J 2000; 76:223-226.

27. Perlmutter D, Suico C, Krauss A, Auld P. A program to reduce discharge delays in a neonatal intensive care unit. Am J Man Care 1998; 4:548-552.

28. Williams T, Leslie G. Delayed discharge from an adult intensive care unit. Aus Health Rev 2004; 28:87-96.

29. Chaboyer W, Foster M, Foster M, Kendall E. The intensive care unit liaison nurse: towards a clear role description. Inten Crit Care Nurs 2004; 20:7-86.
30. Coad S, Haines S. Supporting staff caring for critically ill patients in acute care areas. Nurs Crit Care 1999; 4:245-248.

31. Ball C, Kirkby M, Williams S. Effect of the critical care outreach team on patient survival to discharge from hospital and readmission to critical care: non-randomised population based study. BMJ 2003; 327:1015-1017.

32. Barbetti J, Choate K. Intensive care unit liaison service: implementation at a major metropolitan hospital. Aust Crit Care 2003; 15:46-52.

33. Coombs M, Dillon A. Crossing boundaries, re-defining care: the role of the critical care outreach team. J Clinical Nursing 2002; 11:387-393.

34. Haines S, Coad S. Supporting ward staff in acute care areas: expanding the service. Intens Crit Care Nurs 2001; 17:105109.

35. Russell S. Reducing admission to the intensive care unit. Heart \& Lung 1999; 28:365-372.

36. Angus DC, Carlet J. Surviving intensive care: a report from the 2002 Brussels roundtable. Intens Care Med 2003; 29:368-377.

37. Australian and New Zealand Intensive Care Society [ANZICS]. ANZICS Research Centre for Critical Care Resources. www. anzics.com.au/admc/registry.htm Accessed 2 October 2003.

38. Cameron P, Scown P, Campbell D. Managing access block. Aust Health Rev 2002; 25:59-68.

39. Richardson D. The access-block effect: relationship between delay to reaching an inpatient bed and inpatient length of stay. Med J Aust 2002; 177:492-495.

40. Richardson D. Responses to access-block in Australia: Australian Capital Territory. Med J Aust 2003; 178:103-104.

41. Department of Health. Comprehensive critical care: a review of adult critical care services. London HMSO; 2002.

42. Leith B. Transfer anxiety in critical care patients and their family members. Crit Care Nurse 1998; 18:24-32.

43. McKinney AA, Deeny P. Leaving the intensive care unit: a phenomenological study of the patients' experience. Intens Crit Care Nurs 2002; 18:320-331.

44. Hall-Smith J, Ball C, Coakley J. Follow-up services and the development of a clinical nurse specialist in intensive care. Intens Crit Care Nurs 1997; 13:243-248.

45. Flynn TN, Whitley E, Peters, TJ. Recruitment strategies in a cluster randomised trial-cost implications. Stat Med 2002; 21:397-505.

46. Kerry SM, Bland MJ. Sample size in cluster randomisation. BMJ 1998; 316:559. 